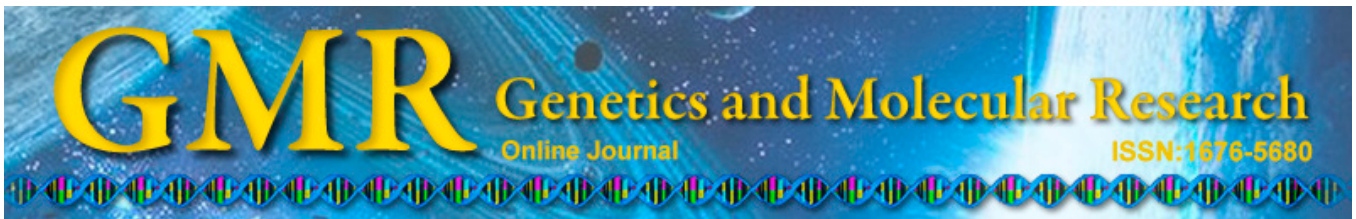

\title{
Technological value of SPECT/CT fusion imaging for the diagnosis of lower gastrointestinal bleeding
}

\author{
Z.G. Wang, G.X. Zhang, S.H. Hao, W.W. Zhang, T. Zhang, \\ Z.P. Zhang and R.X. Wu \\ Department of Nuclear Medicine, \\ General Hospital of Shenyang Military Area Command, Shenyang, China \\ Corresponding author: G.X. Zhang \\ E-mail: guoxuzhangen@163.com
}

Genet. Mol. Res. 14 (4): 14947-14955 (2015)

Received July 17, 2015

Accepted September 28, 2015

Published November 24, 2015

DOI http://dx.doi.org/10.4238/2015.November.24.2

\begin{abstract}
The aim of this study was to assess the clinical value of diagnosing and locating lower gastrointestinal (GI) bleeding using single photon emission computed tomography (SPECT)/computed tomography (CT) fusion imaging with $99 \mathrm{mTc}$ labeled red blood cells

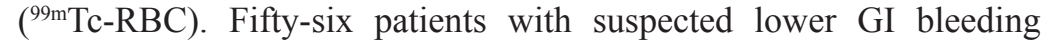
received a preoperative intravenous injection of ${ }^{99 \mathrm{~m}} \mathrm{Tc}-\mathrm{RBC}$ and each underwent planar, SPECT/CT imaging of the lower abdominal region. The location and path of lower GI bleeding were diagnosed by contrastive analysis of planar and SPECT/CT fusion imaging. Among the 56 patients selected, there were abnormalities in concentrated radionuclide activity with planar imaging in 50 patients and in SPECT/ CT fusion imaging in 52 patients. Moreover, bleeding points that were coincident with the surgical results were evident with planar imaging in 31 patients and with SPECT/CT fusion imaging in 48 patients. The diagnostic sensitivity of planar imaging and SPECT/CT fusion imaging were $89.3 \%(50 / 56)$ and $92.9 \%(52 / 56)$, respectively, and the difference
\end{abstract}


was not statistically significant $\left(\chi^{2}=0.11, \mathrm{P}>0.05\right)$. The corresponding positional accuracy values were $73.8 \%(31 / 42)$ and $92.3 \%(48 / 52)$, and the difference was statistically significant $\left(\chi^{2}=4.63, \mathrm{P}<0.05\right) .{ }^{99 \mathrm{~m}} \mathrm{Tc}-$ $\mathrm{RBC}$ SPECT/CT fusion imaging is an effective, simple, and accurate method that can be used for diagnosing and locating lower GI bleeding.

Key words: Lower gastrointestinal bleeding; ${ }^{99 \mathrm{~m} T c-R B C ; ~ T o m o g r a p h y ; ~}$ Emission-computed; Single-photon

\section{INTRODUCTION}

Lower gastrointestinal (GI) bleeding refers to hemorrhage or blood loss in the colon and rectum (Weldon et al., 2008; Okazaki et al., 2009). Colon diverticulitis and angiodysplasia (including varication) are among the main causes of lower GI bleeding. Although more than $80 \%$ of bleeding is caused by angiodysplasia and $75 \%$ by diverticular hemorrhage, bleeding can cease spontaneously in some patients (Lee and Laberge, 2004; Lim and Ahmed, 2004); however, there is a chance of bleeding recurrence if the patients remain untreated. The mismanagement of such cases can not only delay treatment but also increase the difficulty of diagnosis and treatment.

Several methods are currently used for lower GI bleeding diagnosis including X-ray barium meal fluoroscopy, fiber optic endoscopy, selective digital subtraction angiography (DSA), and abdominal laparotomy. Some methods, however, cannot locate the hemorrhage site accurately due to anatomical features, as well as diversity and complexity in causal factors. Some cases may not even be diagnosed during surgery, thus resulting in post-operative bleeding recurrence. Therefore, confirmation of the hemorrhage site is essential for treatment and prognosis. The positive percentage of acute GI bleeding and vasculopathy diagnosis by $\mathrm{X}$-ray GI imaging, such as barium meal, barium enema, and pneumobarium double-contrast imaging, is low. The diagnosis rate of pneumobarium double-contrast imaging is approximately 25 to $60 \%$. When using fiber optic endoscopy for acute massive hemorrhages, the large amount of blood present can obscure visual detection; thus, identifying the location of the hemorrhage site can be difficult. Fiber optic endoscopy is also not particularly advantageous for locating hemorrhage sites that are far from the checkpoint or for locating unobvious vasculopathy during bleeding cessation (Yu et al., 2006). DSA, on the other hand, has a high hemorrhage site detection rate and can diagnose bleeding, occurring at a rate of as low as 0.5 $\mathrm{mL} / \mathrm{min}$, in 50 to $72 \%$ of patients. Super-selective arteriography also has a high diagnostic accuracy for the detection of intestinal tumors and vasculopathies, and the accuracy of this procedure in the active bleeding phase is approximately $90 \%$ (Lu et al., 2001). However, DSA is an invasive technique (Wu et al., 2007) that can be intolerable for patients with serious conditions; therefore, DSA is unsuitable for repeat examinations, as well as in patients with combined coagulation disorders or renal inadequacy, or those who are allergic to contrast medium. Exploratory laparotomy can also exhibit a certain degree of inaccuracy in detecting GI bleeds, and patients are unlikely to consent to this procedure. Therefore, there is a still a need for a more safe, convenient, and highly efficient detection method. Single photon emission computed tomography (SPECT)/computed tomography (CT) fusion imaging is a non-invasive, simple, convenient, and safe method that can support positioning diagnosis for unexplained lower GI bleeding. This article provides a retrospective analysis of lower GI bleeding SPECT/ CT fusion imaging in 56 patients from 2003 to 2011. 


\section{MATERIAL AND METHODS}

\section{Clinical data}

This study included 56 patients ( 42 males and 14 females) between the ages of 14 and 76 years. All study patients were admitted to the General Hospital of the Shenyang Military Region (Shenyang, China) between 2003 and 2011. Ethical approval was sought and received from the hospital ethics committee, and all study-related procedures were conducted in accordance with the tenets of the Declaration of Helsinki. Written informed consent was also obtained from each participant. Clinical symptoms of the study patients included hemafecia, tarry stool, bright red or dull red stool, and bleeding of approximately $45 \mathrm{~mL} / \mathrm{d}$ to $1200 \mathrm{~mL} / \mathrm{d}$. The courses of disease among the patients varied from 1 day to 6 years and 3 months on average. Of the 56 patients, 12 presented with blood shock and 5 had a history of GI surgery. Fibercolonoscope checks conducted before surgery showed no abnormalities. All the patients underwent SPECT/CT fusion imaging with $99 \mathrm{mTc}$ labeled red blood cells $\left({ }^{99 \mathrm{~m}} \mathrm{Tc}-\mathrm{RBC}\right)$ for diagnosis and localization; 48 patients proceeded with exploratory laparotomy to identify the bleeding focus; 6 patients opted for fibercolonoscopy for hemostatic therapy according to the positioning diagnosis results, and 2 patients self-recovered with conservative treatment.

\section{Preparations}

Patients were administered $400 \mathrm{mg} \mathrm{KClO}_{4}$ approximately $1 \mathrm{~h}$ prior to scans to close the gastric mucosa.

\section{Imaging agent}

In this study, an in vivo labeled red blood cell (RBC) method was used. Patients were injected with stannous pyrophosphate intravenously by 0.5 to $1 \mathrm{~mL}$ injection, $30 \mathrm{~min}$ following the oral administration of $400 \mathrm{mg} \mathrm{KClO}_{4}$. Patients then assumed the supine position, and after $15 \mathrm{~min}$, they were administered ${ }^{99 \mathrm{~m}} \mathrm{TCO}_{4}^{-}$elutriant $370 \mathrm{MBq}(10 \mathrm{mci})$ by intravenous injection.

\section{Imaging methods}

The equipment used in this study was U.S. GE VG-Hawkeye multi-functional double probes SPECT/CT (GE Healthcare, VI, USA) with the following specifications: low energy, general parallel aperture collimator, energy peak at $140 \mathrm{keV}$, window width of $20 \%$, and matrix of $128 \times 128$. The probe was adjusted to the abdomen (from the processus xiphoideus to pubic symphysis), and the patient assumed the supine position. Patients were then intravenously administered ${ }^{99 \mathrm{~m}} \mathrm{TCO}_{4}^{-}$elutriant $370 \mathrm{MBq}(10 \mathrm{mci})$, and initially, 30 frames were acquired consecutively for blood perfusion phase dynamic imaging at a rate of $3 \mathrm{~s} /$ frame, following which 60 frames were acquired consecutively for blood pool phase dynamic imaging at a rate of $1 \mathrm{~s} /$ frame. Patients who still showed negative results after 60 min were required to undergo delayed imaging at 2,4 , and $8 \mathrm{~h}$, or even at $24 \mathrm{~h}$ if necessary. When the planar imaging abnormality ended, nuclide tomography, CT tomography, and imaging fusion were conducted. The results were expressed either as positive or negative. The determination criteria of a positive result were the presence of abnormal radionuclide concentration foci in 
the abdomen on multi-frame images, in which the local radioactive distribution concentrated gradually by time; the followed intestinal canal began to image successively, and the bleeding site could be determined as the earliest abnormal radioactive foci. A negative result indicated that no bleeding or slight bleeding was detected during the procedure.

\section{Data processing}

All statistical analyses were performed using the SPSS 10.1 software. Chi-square $\left(\chi^{2}\right)$ analysis was used for both planar imaging and fusion imaging data. $\mathrm{P}<0.05$ was considered to be significantly different.

\section{RESULTS}

Among the 56 cases, lower abdominal, abnormal radioactive concentrated loci were detected in 50 planar images and 52 fusion images. Fusion imaging diagnosis located bleeding in the ileocecum, terminal ileum, flexura hepatica coli, transverse colon, and flexura lienalis coli in $28,13,6,2$, and 3 patients, respectively. Images from a 51-year-old male and 10-year-old male included in the study are provided in Figure 1 and Figure 2, respectively. Of these 52 patients, the positioning diagnostic results were coincident with the surgical results in 48 patients. In the other 4 patients, the procedure had to be terminated due to abdominal pain during imaging, and the results of these patients were considered negative. Planar imaging located bleeding in the ileocecum, terminal ileum, flexura hepatica coli, and flexura lienalis coli in 26, 11, 4, and 1 patients, respectively. However, bleeding in 8 other patients could not be located due to unrecognized anatomical sites. Of these 42 patients, the results were coincident with the surgical results in 31 patients. The diagnostic sensitivity of planar imaging and SPECT/CT fusion imaging were $89.3 \%$ $(50 / 56)$ and $92.9 \%(52 / 56)$, respectively, and the difference was not statistically significant $\left(\chi^{2}\right.$ $=0.11, \mathrm{P}>0.05)$. The corresponding positional accuracy values were $73.8 \%(31 / 42)$ and $92.3 \%$ (48/52), and the difference was considered to be statistically significant $\left(\chi^{2}=4.63, \mathrm{P}<0.05\right)$.

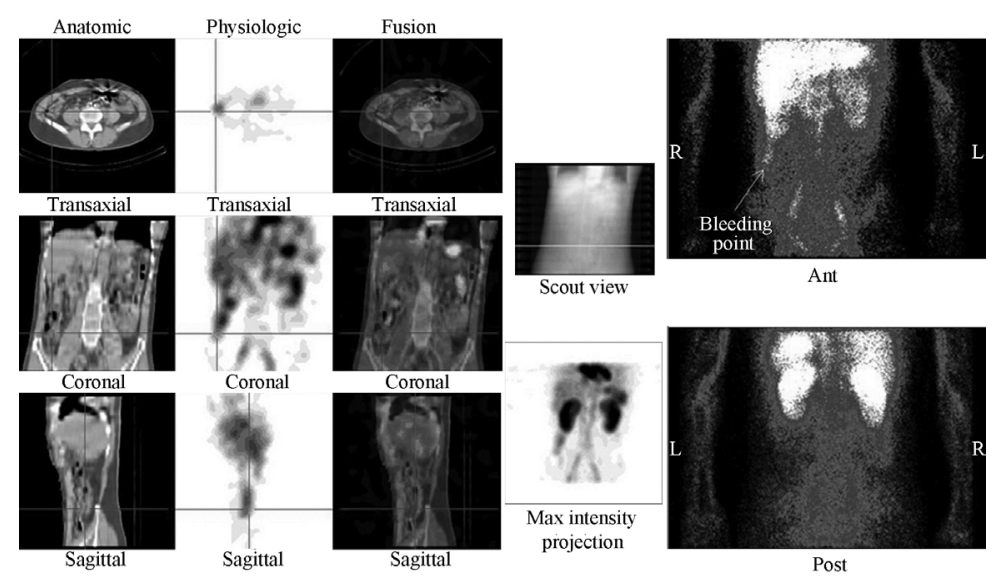

Figure 1. Male, 51 years old, fecal occult blood showed positive, no bleeding point observed during initial fiber colonoscopy or with repeat examination, in ${ }^{99 \mathrm{~m}} \mathrm{Tc}-\mathrm{RBC}$ SPECT/CT fusion positioning imaging, ${ }^{99 \mathrm{~m} T c-R B C}$ effused from middle and lower segments of the ascending colon near the ileocecal junction and formed column shape abnormal nuclide concentrated foci which suggested bleeding in this region, and was confirmed during surgery the following day. 

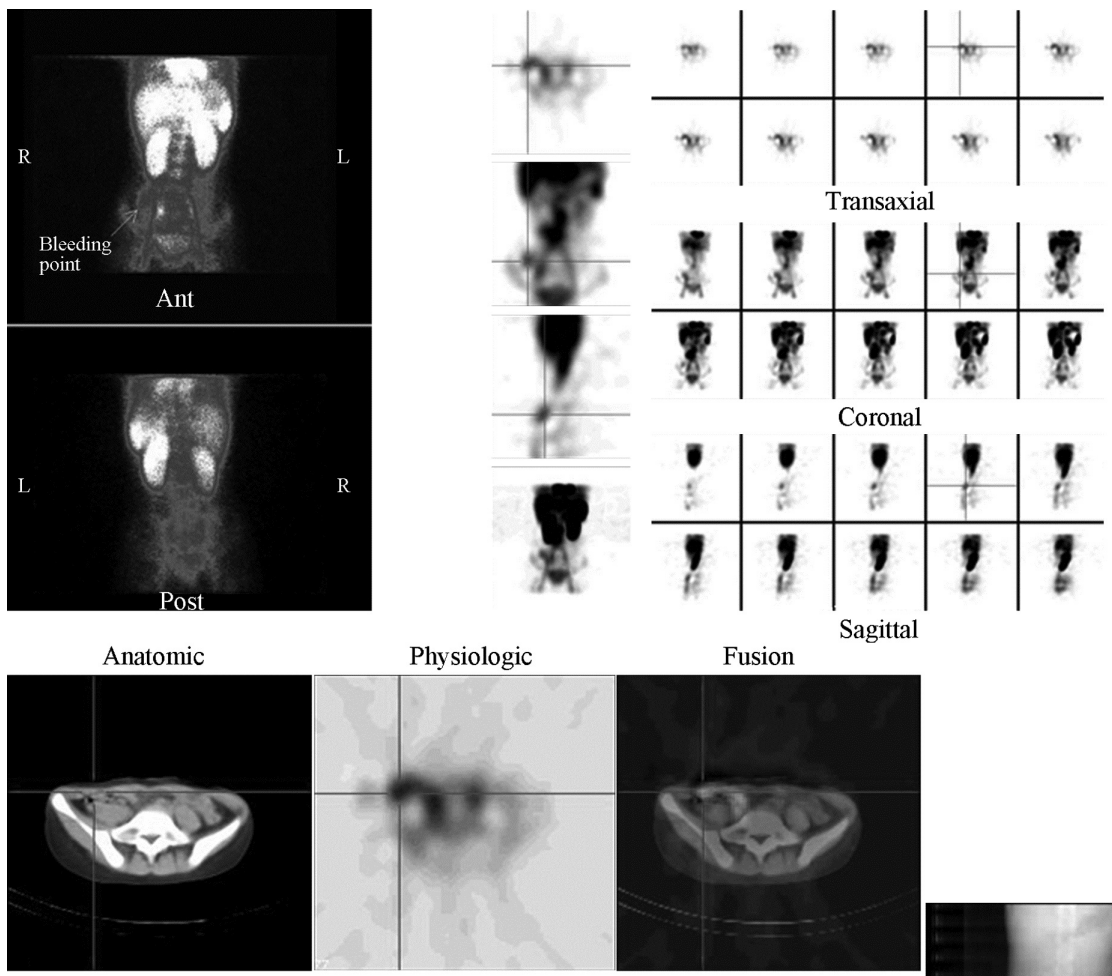

Physiologic

Fusion

Sagittal
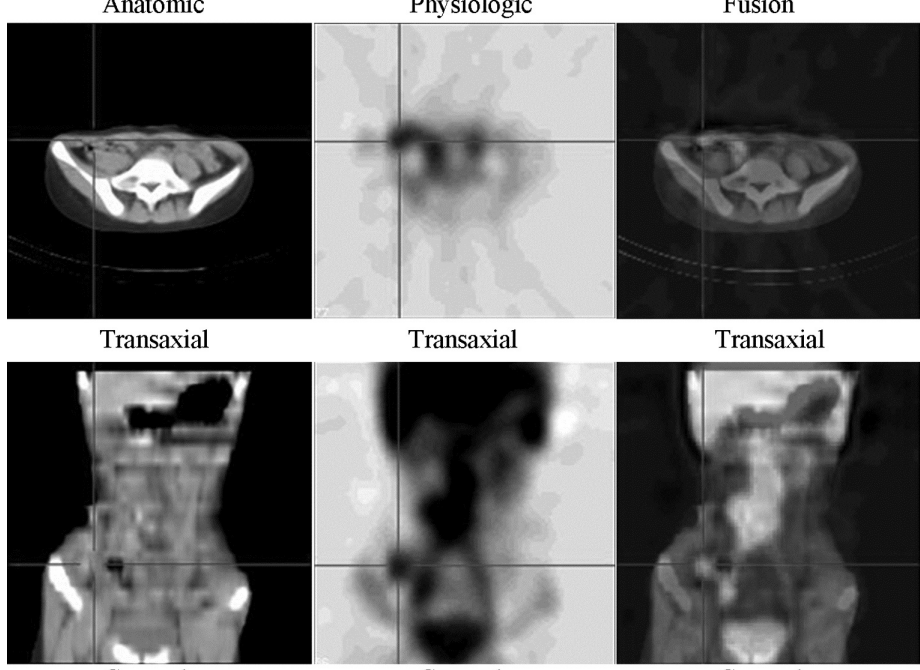

Transaxial

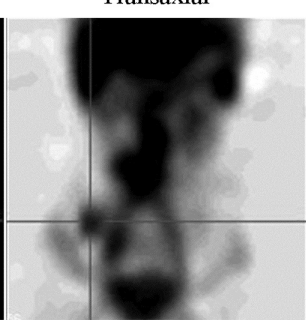

Coronal
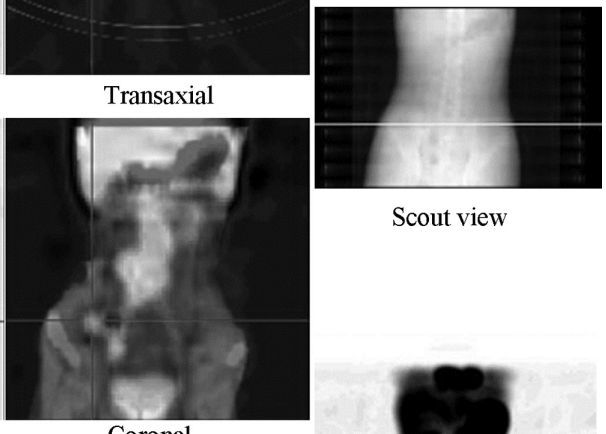

Scout view
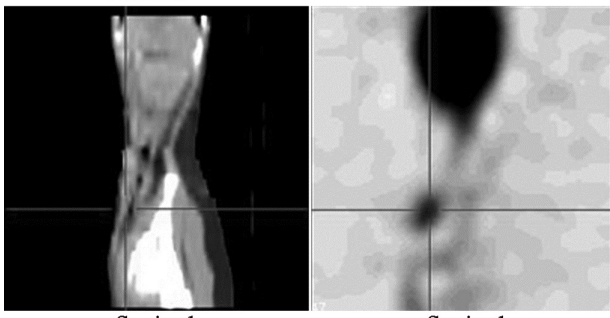

Sagittal

Coronal

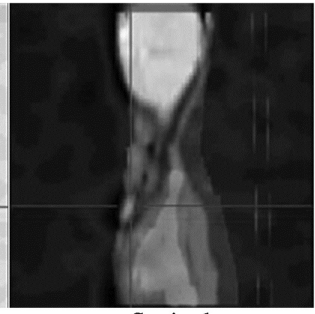

Sagittal

Figure 2. SPECT/CT fusion imaging of the lower abdomen in a 10 -year-old male patient. The patient suffered from intermittent blood in stool for 4 years and was admitted due to persistent complications for 3 days, including anemia. No obvious abnormalities were observed during ultrasound of the abdominal and pelvic cavity. ${ }^{99 \mathrm{~m}} \mathrm{Tc}-\mathrm{RBC}$ effused from middle and lower segments of ascending colon near the ileocecal junction and formed abnormal column-shape nuclide concentrated foci, suggesting the bleeding site in this region. This was later confirmed by surgery during the following day. 


\section{DISCUSSION}

This study assessed the clinical value of SPECT/CT fusion imaging for diagnosing and determining lower GI bleeding, which refers to bleeding under the Treitz ligament. The main causes of this condition include intestinal malignant tumors, polyposis intestinalis, Meckel's diverticulum, vascular malformation, inflammatory bowel disease, and trauma. Routine examinations may be limited or hindered by patient-based factors, as well as individual variability with regards to characteristics of the lower digestive tract. Although most patients will stop bleeding spontaneously without undergoing any specific intervention, the clinical condition of these patients can be potentially life-threatening particularly when there is clinical evidence of blood volume loss from an indeterminate site (Suzman et al., 1996). Viable therapeutic results can be achieved when a multi-disciplinary approach is pursued (Vernava et al., 1997). In a majority of patients, the site of the hemorrhage can be localized and managed endoscopically. In a variable percentage of cases, however, the source of bleeding remains unknown, and these patients may need to undergo emergency surgical resection (Anthony et al., 2007).

Localization of the hemorrhage site before treatment is therefore of the utmost importance to avoid blind resection, which is associated with a high rate of morbidity and mortality (Rockey, 2006). In particular, the source of a lower GI hemorrhage can often be difficult to localize accurately, and clinicians still disagree over which is the most reliable diagnostic test to use (O'Neill et al., 2000). For instance, endoscopy, angiography, multi-slice CT, capsular endoscopy, and RBC scintigraphy all have distinct advantages and disadvantages for the diagnosis of bleeding in the GI tract.

Endoscopy has the potential benefit of being both diagnostic and therapeutic. Nevertheless, bleeding sites can be difficult to visualize during severe hemorrhaging, even by the most skilled operator. Intermittent GI bleeding can also lead to inaccurate diagnosis. Colonoscopy is unable to detect bleeding in the small intestine (with the exception of the ileal region) and is relatively invasive, with risks that include bowel perforation, exacerbation of bleeding, and septicemia ( $\mathrm{Lu}$ et al., 2001). Endoscopic examination of the colon is also often difficult when active bleeding may obscure the presence of diverticula, angiodysplasia, patchy colitis, or small polyps (Strate and Syngal, 2005).

Angiography offers some therapeutic options, such as arterial embolization. However, the capacity of this procedure to detect a hemorrhagic site is limited when the bleeding rate is less than $1.0 \mathrm{~mL} / \mathrm{min}$ (Howarth, 2006). Similar to colonoscopy, angiography can be nondiagnostic when GI blood loss is intermittent or quiescent. This procedure requires active bleeding not only at the time of the examination but also at the time of contrast medium injection. The accuracy of this procedure in detecting the site of blood loss ranges from 43 to $87 \%$ (Lim and Ahmed, 2004). Angiography is also invasive with a $\%$ rate of complications including contrast-induced renal failure, arterial injury and/or thrombosis, and mesenteric ischemia (Peter and Dougherty, 1999).

Multi-slice CT angiography is sensitive in localizing extravasated intravenous contrast medium within the bowel lumen, but this procedure still requires active bleeding at the time of imaging. Nevertheless, even if an active bleeding site is not determined, multi-slice CT angiography can still identify pathological lesions (Gupta and Reddy, 2007). On the other hand, capsule endoscopy is non-invasive and generally does not require significant bowel preparation. This procedure allows non-invasive imaging of the entire small bowel and is currently used in the out-patient setting, and when a history of obscure bleeding is present 
(Gupta and Reddy, 2007). However, accumulating evidence suggests that capsule endoscopy during, or as close as possible to, the active bleeding event may reveal the bleeding source in up to $92 \%$ of patients (Apostolopoulos et al., 2007). The main contraindication is a known or suspected GI obstruction, strictures, or fistulae. These conditions may lead to capsule retention and possible surgery if the capsule is not excreted naturally. Other contraindications are difficulty in swallowing, large or numerous intestinal diverticula in which the capsule might become lodged, Zenker's diverticulum, extensive Crohn's disease, prior pelvic or abdominal surgery, pregnancy, and the presence of implanted electronic devices such as pacemakers (Hartmann et al., 2004). In approximately $20 \%$ of patients, the capsule batteries have also been reported to wear out before the device reaches the cecum (van Tuyl et al., 2004).

Nuclear medicine techniques have served a role in this clinical scenario for about 30 years with two radionuclide imaging techniques, namely, ${ }^{99 \mathrm{~m}} \mathrm{Tc}-\mathrm{RBC}$ and ${ }^{99 \mathrm{~m}} \mathrm{Tc}-$ labelled sulfur colloid. These techniques are used for the localization of acute GI bleeding (Zuckier, 2003). A clinical suspicion of bleeding is attributable to a Meckel's diverticulum, and ${ }^{99 \mathrm{~m}} \mathrm{Tc}-$ pertechnetate imaging can be acquired to confirm or exclude the presence of ectopic gastric mucosa (Ford et al., 1999). Currently, radionuclide bleeding scintigraphy is most commonly performed using the ${ }^{99 \mathrm{~m}} \mathrm{Tc}-\mathrm{RBC}$ planar scan, which can detect the site of blood loss even at a minimal blood flow of $0.05 \mathrm{~mL} / \mathrm{min}$ (Sgourakis et al., 2011). A positive result can be acquired, and the coincidence rate is $85 \%$ (Galbois et al., 2012). This technique is simple, safe, and non-invasive and even allows for continuous monitoring over hours, which is a major advantage in comparison to other diagnostic methods because most GI bleeds are intermittent and thus frequently missed (Marignani et al., 2005). Accuracy in the localization of the bleeding site can be improved by acquiring a continuous dynamic scan that can detect RBC accumulation as early as possible (Meller et al., 2000). However, some possible limitations should be considered with regard to establishing a diagnosis of GI bleeding, and particularly in attempting to localize the bleeding source. The main limitation of RBC planar imaging is the inadequacy of anatomical information that this procedure provides, which can then preclude the correct identification of the site of GI bleeding (Howarth et al., 2002). The strong peristaltic and anti-peristaltic movements produced by the irritative effect of blood on the bowel wall may also preclude correct identification of the bleeding site. These movements can push RBCs within the lumen from the bleeding site to another location where the RBCs are later detected by scintigraphy.

SPECT/CT fusion imaging can improve the identification of anatomical sites because of the true 3D nature of the information acquired, which is typically presented as cross-sectional slices through the patient. Fusion imaging, which combines functional data derived from SPECT with the morphological details yielded by CT or magnetic resonance imaging, can facilitate the identification of the source of bleeding by providing accurate anatomical information about the sites of ${ }^{99 \mathrm{~m}} \mathrm{Tc}-\mathrm{RBC}$ accumulation. Moreover, the recent introduction of hybrid SPECT/CT cameras has made the process of image fusion easier and more reliable. These systems can acquire SPECT and CT images in the same session with images acquired simultaneously by means of the hardware (O'Connor and Kemp, 2006). To date, the vast majority of applications of this technique have been reported in oncology. Nevertheless, SPECT/ CT can also be used successfully in patients with other diseases (Schillaci et al., 2004; Bunyaviroch et al., 2006; Filippi and Schillaci, 2006).

In this study, bleeding could not be located accurately in 8 cases due to the presence of unrecognized anatomical sites. SPECT/CT fusion imaging not only generates 3D nuclide images but can also combine nuclide images with CT images, which is an obvious advantage. 
Aside from locating the bleeding site accurately, CT can also detect the cause of lower GI bleeding, which is essential. No statistical significance of diagnostic sensitivity existed between planar imaging and SPECT/CT fusion imaging. However, the corresponding positional accuracy value of SPECT/CT was significantly higher than planar imaging. SPECT/CT fusion imaging is a combination of two imaging technologies with certain diagnostic advantages, including the high detection accuracy of SPECT and the clear anatomical relationships illustrated by CT. This method may compensate for the poor accuracy of emission computed tomography positioning. Our findings provide a reliable basis for operative treatment.

\section{Conflicts of interest}

The authors declare no conflict of interest.

\section{REFERENCES}

Anthony S, Milburn S and Uberoi R (2007). Multi-detector CT: review of its use in acute GI haemorrhage. Clin. Radiol. 62: 938-949.

Apostolopoulos P, Liatsos C, Gralnek IM, Kalantzis C, et al. (2007). Evaluation of capsule endoscopy in active, mild-tomoderate, overt, obscure GI bleeding. Gastrointest. Endosc. 66: 1174-1181.

Bunyaviroch T, Aggarwal A and Oates ME (2006). Optimized scintigraphic evaluation of infection and inflammation: role of single-photon emission computed tomography/computed tomography fusion imaging. Semin. Nucl. Med. 36: 295-311.

Filippi L and Schillaci O (2006). SPECT/CT with a hybrid camera: a new imaging modality for the functional anatomical mapping of infections. Expert. Rev. Med. Devices 3: 699-703.

Ford PV, Bartold SP, Fink-Bennett DM, Jolles PR, et al. (1999). Procedure guideline for gastrointestinal bleeding and Meckel's diverticulum scintigraphy. J. Nucl. Med. 40: 1226-1232.

Galbois A, Stenard F and Margetis D (2012). A ghost pancreas. Gastroenterology 142: 211, 412.

Gupta R and Reddy DN (2007). Capsule endoscopy: current status in obscure gastrointestinal bleeding. World $J$. Gastroenterol. 13: 4551-4553.

Hartmann D, Schilling D, Bolz G and Riemann JF (2004). Capsule endoscopy, technical impact, benefits and limitations. Langenbecks. Arch. Surg. 389: 225-233.

Howarth DM (2006). The role of nuclear medicine in the detection of acute gastrointestinal bleeding. Semin. Nucl. Med. 36: $133-146$.

Howarth DM, Tang K and Lees W (2002). The clinical utility of nuclear medicine imaging for the detection of occult gastrointestinal haemorrhage. Nucl. Med. Commun. 23: 591-594.

Lee EW and Laberge JM (2004). Differential diagnosis of gastrointestinal bleeding. Tech. Vasc. Interv. Radiol. 7: 112-122.

Lim JK and Ahmed A (2004). Endoscopic approach to the treatment of gastrointestinal bleeding. Tech. Vasc. Interv. Radiol. 7: 123-129.

Lu WS, Huang ML, Yang SH, Fei ZJ, et al. (2001). The value of angiography of gastrointestinal bleeding and interventional treatment. J. Interventional Radioactive Sci. 10: 138-140.

Marignani M, Angeletti S, Filippi L, Danieli R, et al. (2005). Occult and obscure bleeding, iron deficiency anemia and other gastrointestinal stories (Review). Int. J. Mol. Med. 15: 129-135.

Meller J, Schönborn E, Conrad M, Horstmann O, et al. (2000). Improved demonstration of gastrointestinal bleeding sites by means of $99 \mathrm{mTc}$-labeled autologous erythrocytes and continuous dynamic scintigraphy with cine-mode display. Chirug 71: 292-299.

O'Connor MK and Kemp BJ (2006). Single-photon emission computed tomography/computed tomography: basic instrumentation and innovations. Semin. Nucl. Med. 36: 258-266.

Okazaki H, Fujiwara Y, Sugimori S, Nagami Y, et al. (2009). Prevalence of mid-gastrointestinal bleeding in patients with acute overt gastrointestinal bleeding; multi-center experience with 1044 consecutive patients. J. Gastroenterol. 44: 550-555.

O’Neill BB, Gosnell JE, Lull RJ, Schecter WP, et al. (2000). Cinematic nuclear scintigraphy reliably directs surgical intervention for patients with gastrointestinal bleeding. Arch. Surg. 135: 1076-1082.

Peter DJ and Dougherty JM (1999). Evaluation of the patient with gastrointestinal bleeding: an evidence based approach. Emerg. Med. Clin. North Am. 17: 239-261. 
Rockey DC (2006). Lower gastrointestinal bleeding. Gastroenterology 130: 165-171.

Schillaci O, Danieli R, Manni C and Simonetti G (2004). Is SPECT/CT with a hybrid camera useful to improve scintigraphic imaging interpretation? Nucl. Med. Commun. 25: 705-710.

Sgourakis G, Kontovounisios C and Karaliotas C (2011). Bleeding Duodenal Mass. Gastroenterology 141: e1-2.

Strate LL and Syngal S (2005). Predictors of utilization of early colonoscopy vs radiography for severe lower intestinal bleeding. Gastrointest. Endosc. 61: 46-52.

Suzman MS, Talmor M, Jennis R, Binkert B, et al. (1996). Accurate localization and surgical management of acute lower gastrointestinal hemorrhage with technetium-labeled erythrocyte scintigraphy. Ann. Surg. 224: 29-36.

van Tuyl SA, Kuipers EJ, Timmer R and Stolk MF (2004). Video capsule endoscopy: procedure, indications and diagnostic yield. Neth. J. Med. 62: 225-228.

Vernava AM 3rd, Moore BA, Longo WE and Johnson FE (1997). Lower gastrointestinal bleeding. Dis. Colon Rectum. 40: 846-858.

Weldon DT, Burke SJ, Sun S, Mimura H, et al. (2008). Interventional management of lower gastrointestinal bleeding. Eur. Radiol. 18: 857-867.

Wu W, Xia HH, Cheng ZG, Liu YS, et al. (2007). Angiography diagnosis and interventional treatment of acute gastrointestinal bleeding. J. Med. Imag. 17: 1333-1335.

Yu JB, Xiang CH and Wang HZ (2006). The effect of combined application of SPECT/CT and intraoperative endoscope in diagnosis positioning for lower gastrointestinal bleeding with unknown reasons. Chin. J. Bases Clin. General. Surg. 13: 573-575.

Zuckier LS (2003). Acute gastrointestinal bleeding. Semin. Nucl. Med. 33: 297-311. 